\title{
Generalized active disturbance rejection control for the boiler-turbine unit using multi-objective optimization and extended state observer
}

\author{
Jianzhong $\mathrm{Zhu}^{1,2}$, Xiao $\mathrm{Wu}^{1}$, Jiong Shen ${ }^{1, *}$, and Meihong Wang ${ }^{3}$ \\ ${ }^{1}$ Key Laboratory of Energy Thermal Conversion and Control of Ministry of Education, School of \\ Energy and Environment, Southeast University, Sipailou 2, Nanjing 210096, China \\ ${ }^{2}$ School of Electric Power Engineering, Nanjing Institute of Technology, Hongjing Road 2, Nanjing \\ 211167, China \\ ${ }^{3}$ Department of Chemical and Biological Engineering, Univerisity of Sheffield, Sheffield S1 3JD, UK
}

\begin{abstract}
This paper proposes a generalized active disturbance rejection controller (GADRC) based hierarchical control structure for the boilerturbine unit. In the lower layer, a multivariable extended state observer (MESO) is developed to estimate the values of the lumped disturbances caused by modelling mismatches, fuel quality variation and wide range load variation. The influence of the disturbances is then compensated at the input side as a feedforward control. In the upper layer, the multi-objective optimization is devised to obtain the set-points by removing the plant behaviour variation from the optimized model in a feasible way. The lowpass filter acting on the lumped disturbances is designed to bridge the gap between the lower and upper layer. The impact of the feedthrough item is approximated by a first-order system and a two degree-of-freedom (2-DOF) control strategy is established to illustrate the set-point tracking and disturbance rejection properties of the controller. Simulation studies on a $1000 \mathrm{MWe}$ coal-fired ultra-supercritical boiler-turbine unit demonstrate that the proposed control strategy can achieve a satisfactory performance in cases of fuel quality variations, model-plant mismatches and wide range load variation.
\end{abstract}

\section{Introduction}

Boiler-turbine units are the core devices in modern fossil fuel fired power plants, in which the energy of the coal is transformed into steam thermal energy by the boiler then to the rotation energy in the turbine and finally to the electrical energy through the synchronous generator. Control of boiler-turbine units is challenging due to its characteristics of severe nonlinearity, interactions between different inputs and outputs, large inertia and time-delays. Moreover, the plant behavior variation and unknown disturbances caused by load varying, fuel quality change, coking and ash deposit in boiler, will degrade the performance of predesigned controller and affect the safe and economical operation of the plant.

\footnotetext{
*Corresponding author: shenj@seu.edu.cn
} 
For such a process plant, the conventional PID is still the most used control strategy. In [1], the author presented a tuning method for the proposed PID controller. To enhance the robustness of the controller, a robust PID has been reported in [2], where the robustness stability was guaranteed. A gain-scheduling method was applied to the boiler-turbine unit in [3] to handle the issue of large nonlinearity due to wide range load variation. The parameters of the controller were scheduled along with the plant behavior change. Although PID is acknowledged as a simple and an efficient method in process control, various constraints are increasingly limiting its application in power plant control [4]. For example, the incidental overshoot appeared in output tracking caused by the delayed coal transmission and variation of coal quality, which may influence the safe operation of the plant.

For these reasons, this paper presents a generalized active disturbance rejection control (GADRC) strategy for the boiler-turbine unit to achieve fast set-point tracking and disturbance rejection performance in the presence of internal uncertainties and external disturbances. The essential idea of multivariable extended state observer (MESO), which can estimate the lumped disturbances without decoupling the model into several single loops is utilized in the GADRC design. The ESO was first proposed by Han in [5], in which the lumped disturbance was treated as an extended state of the system and the control system was designed by reducing the plant into an integral chain form. Li et al. [6] presented a generalized extended state observer based control (GESOBC), so that the application of ESO can be extended to the MIMO system. However, the GESOBC approach is not suited for the boiler-turbine unit because the turbine governor valve has a very quick influence on the power output. This feature will bring in difficulties that the disturbances caused by the direct feedthrough input (turbine throttle valve) cannot be compensated. In this paper, the feedthrough part is approximated to a simple first-order dynamic, where the approximate error is regard as a disturbance which can be observed by the MESO and then compensated.

In addition to set-point tracking, attain an optimal operation considering multiobjectives such as energy conservation, safety operation etc. is also desired for the boiler turbine unit. In [7], the particle swarm optimization (PSO) algorithm was introduced into the power plant to optimize the set-points in an off-line mode. However, the method highly depends on the mathematical model. To overcome the issue, the authors in [8] introduced the estimated disturbances into the expression of the multi-objective optimization at each sampling time. One limitation of that method is that the high-frequency fluctuations caused from the external disturbance may draw the set-points into a wrong way or make the optimization infeasible. To be more practical, a low-pass filter should be added on the estimated lumped disturbance, so that the multi-objective optimization is insensitive of high-frequency fluctuations and the solution of the optimization is more feasible.

\section{Problem formulation}

The model under consideration is established based an 1000MWe coal-fired super-critical boiler-turbine unit of Taizhou Power Generation Co, Ltd in China [9]. The model structure is derived from mechanism modeling and the parameters are determined through identifications using operating data. Its dynamics are in summarized as follows, the details of which can be seen from [9]. 


$$
\begin{aligned}
\dot{x}_{1}= & -0.0056 x_{1}+0.0056 e^{-17 s} u_{1} \\
\dot{x}_{2}= & \frac{\left(43.22 x_{2}-5.62 x_{2}^{0.882}-31.84\right)\left(-8.96 x_{2}+1.165 x_{2}^{0.882}+2512.4\right)\left(500-1.31 x_{3}\right)}{1060000\left(1.31 x_{3}-1205\right)} u_{3} \\
& +0.0157\left(x_{1}\right)^{1.031}+0.000665 u_{2} \\
\dot{x}_{3}= & \frac{\left(43.22 x_{2}-5.62 x_{2}^{0.882}-31.84\right)\left(-8.96 x_{2}+1.165 x_{2}^{0.882}+2512.4\right)\left(3000-1.31 x_{3}\right)}{59830\left(1.31 x_{3}-1205\right)} u_{3} \\
& +0.278\left(x_{1}\right)^{1.031}-0.03 u_{2} \\
y_{1}= & x_{2}-0.13 x_{2}^{0.882} \\
y_{2}= & x_{3} \\
y_{3}= & 0.00055 u_{3}\left(43.22 x_{2}-5.62 x_{2}^{0.882}-31.84\right)\left(-8.96 x_{2}+1.165 x_{2}^{0.882}+2512.4\right)
\end{aligned}
$$

where the state variables are pulverized coal flow rate that feeds the boiler, $r_{B}$ in $\mathrm{kg} / \mathrm{s}$, represented as $x_{1}$, separator steam pressure, $p_{m}$ in $M P a$, represented as $x_{2}$, and separator enthalpy, $h_{m}$ in $k J / k g$, represented as $x_{3}$; the manipulated variables are fuel flow rate command, $u_{B}$ in $\mathrm{kg} / \mathrm{s}$, represented as $u_{1}$, feedwater flow rate, $D_{f w}$ in $\mathrm{kg} / \mathrm{s}$, represented as $u_{2}$, and turbine throttle valve opening, $u_{t}$ in \%, represented as $u_{3}$ and the controlled variables are throttle steam pressure, $p_{s t}$ in $M P a$, represented as $y_{1}$, and electric power output, $E$ in $M W e$, represented as $y_{3}$.

Table 1. Typical operating points of the boiler turbine unit.

\begin{tabular}{|c|c|c|c|c|c|c|}
\hline & $\# 1$ & $\# 2$ & $\# 3$ & $\# 4$ & $\# 5$ & $\# 6$ \\
\hline$y_{1}(\mathrm{MPa})$ & 14.1 & 17.2 & $\mathbf{1 8 . 3}$ & 22.2 & 23.1 & 24.9 \\
\hline$y_{2}(\mathrm{~kJ} / \mathrm{kg})$ & 2817.0 & 2793.2 & $\mathbf{2 7 2 6 . 3}$ & 2679.0 & 2682.0 & 2686.0 \\
\hline$y_{3}(\mathrm{MWe})$ & 500.0 & 608.3 & $\mathbf{7 3 1 . 9}$ & 800.6 & 904.0 & 1000.0 \\
\hline$u_{1}(\mathrm{~kg} / \mathrm{s})$ & 48.4 & 58.6 & $\mathbf{7 0 . 1}$ & 76.5 & 86.0 & 94.9 \\
\hline$u_{2}(\mathrm{~kg} / \mathrm{s})$ & 365.6 & 450.5 & $\mathbf{5 6 2 . 1}$ & 631.4 & 711.7 & 785.5 \\
\hline$u_{3}(\%)$ & 66.0 & 65.9 & $\mathbf{7 4 . 6}$ & 67.9 & 73.8 & 76.1 \\
\hline
\end{tabular}

Among the typical operating points shown in table 1, the intermediate point, \#3 is chosen using the method of Taylor's series expansion approach to achieve the nominal model $\left(A_{n}, B_{n}, C_{n}, D_{n}\right)$.To further study the control of boiler-turbine in the presence of unknown disturbances, a more generalized dynamic model (2) is used:

$$
\begin{aligned}
& \dot{x}=A_{n} x+B_{n} u+B_{d} f(x, \omega(t), t) \\
& y=C_{n} x+D_{n} u
\end{aligned}
$$

where $x \in R^{n}$ is the state vector, $u \in R^{m}$ is the control input vector, $y \in R^{p}$ is the controlled output vector, $f(x, \omega(t), t) \in R^{q}$ is the lumped disturbance term. To be more general, $B_{d}$ can be set to $I_{n \times n}$. 


\section{Control design}

The working principle of the proposed GADRC is illustrated in figure 1, in which the disturbance compensation channel describes that the lumped disturbances are estimated by the MESO and then actively compensated by an appropriate gain $K_{d}$. The remaining part of the lower layer denoted as feedback channel can be designed from the nominal model to guarantee the stability of the system. The unit load command $E_{u l d}$ is the only input of the upper layer in which the set-points $r$ can be obtained by the multi-objective optimization.

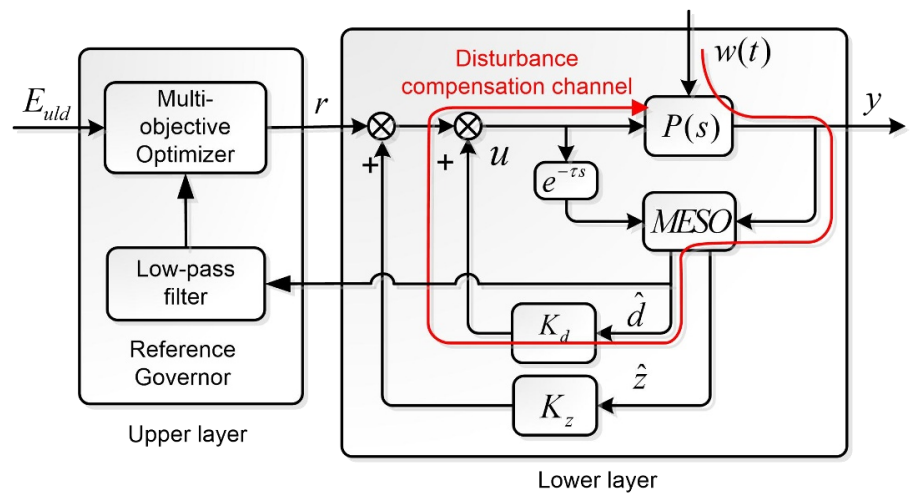

Fig. 1. Overall scheme of the proposed control system.

Remark 1: we can see that the time-delay can be managed independent of the MESO. Due to the modelling error, the time delay parameter $\tau$ should be set as an estimated value, that is $\tau=15$.

\subsection{MESO design}

A modified MESO is developed in this section for the boiler-turbine unit to handle the effect of direct feedthrough, where the influence of the feedthrough can be estimated by taking it as an augmented state $\theta$. The direct feedthrough term $D_{n}$ matrix is approximated by a first order system $\mathcal{D}(s)=D_{n} /(1+\varepsilon s)$, extending the system (2) into an augmented form (3):

$$
\begin{aligned}
& \dot{z}=A_{z} z+B_{z} u+f(z, \omega(t), t) \\
& y=C_{z} z
\end{aligned}
$$

where $z=\left[\begin{array}{ll}x^{T} & \theta^{T}\end{array}\right]^{T} \in R^{n+1}$ is the augmented state vector, $f(z, \omega(t), t)$ is the disturbance term including the approximation error and matrices,

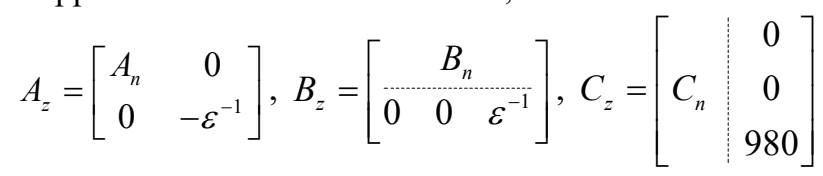

To ensure a fast decay of the augmented state $\theta$, it is necessary to tune the value $\varepsilon$ as small as possible, $\varepsilon=0.4 \mathrm{~s}$. An extended observer can then be devised to estimate the values of the states and the lumped disturbances $d=f(z, \omega(t), t) \in R^{n+1}$ : 


$$
\begin{aligned}
& \dot{\bar{z}}=\bar{A}_{z} \hat{\bar{z}}+\bar{B}_{z} u+L(y-\hat{y}) \\
& \hat{y}=\bar{C}_{z} \hat{\bar{z}}
\end{aligned}
$$

where the variable $\bar{z}=\left[\begin{array}{ll}z^{T} & d^{T}\end{array}\right]^{T}, \hat{\bar{z}}$ is the estimation of $\bar{z}, L$ is the observer gain and matrices,

$$
\bar{A}_{z}=\left[\begin{array}{cc}
A_{z} & B_{d} \\
0 & 0
\end{array}\right], \bar{B}_{z}=\left[\begin{array}{c}
B_{z} \\
0
\end{array}\right], \bar{C}_{z}=\left[\begin{array}{ll}
C_{z} & 0
\end{array}\right]
$$

\subsection{Controller structure}

To achieve a fast output tracking and disturbance rejection performance, the control law is formulated by the combination of linear feedback controls calculated from the state and lumped disturbance estimations and feedforward control calculated from the reference:

$$
u=K_{z} \hat{z}+K_{d} \hat{d}+F_{r} r
$$

The control law can be illustrated from figure 1, in which the disturbance compensation channel including the disturbance compensation gain $K_{d}$ can reduce the plant into the nominal model. The feedback gain $K_{z}$ is designed to stabilize the nominal model $\left(A_{z}, B_{z}\right)$, which can be determined by using the method of pole placement to make $A_{z}+B_{z} K_{z}$ a Hurwitz matrix. The poles of observer should then be placed on the left plane of that for $K_{z}$. The feedforward gain $F_{r}$ is designed for offset free tracking, which can be determined in the subsequent derivation.

Substituting the control law (5) into (4), the dynamics of the controller are obtained by the following equations

$$
\left[\begin{array}{c}
\dot{\hat{z}} \\
\dot{\hat{d}}
\end{array}\right]=\left[\begin{array}{cc}
A_{z}+B_{z} K_{z}-L_{z} C_{z} & B_{d}+B_{z} K_{d} \\
-L_{d} C_{z} & 0
\end{array}\right]\left[\begin{array}{l}
\hat{z} \\
\hat{d}
\end{array}\right]+\left[\begin{array}{c}
L_{z} \\
L_{d}
\end{array}\right] y+\left[\begin{array}{c}
B_{z} F_{r} \\
0
\end{array}\right] r
$$

where $L_{z}, L_{d}$ corresponding to $\hat{z}, \hat{d}$ are the decomposition of the observer gain, i.e., $L=\left[L_{z}^{T}, L_{d}^{T}\right]^{T}$.

Suppose that the control system (6) is in steady-state, then

$$
\left(A_{z}+B_{z} K_{z}-L_{z} C_{z}\right) \hat{z}+\left(B_{d}+B_{z} K_{d}\right) \hat{d}+L_{z} y+B_{z} F_{r} r=0, \quad L_{d}\left(y-C_{z} \hat{z}\right)=0
$$

Due to the stabilization of the observer, $L_{d}$ is invertible, which implies $y=C_{z} \hat{z}$. From the above steady-state equations, we have

$$
y=-C_{z} M\left(B_{d}+B_{z} K_{d}\right) \hat{d}-C_{z} M B_{z} F_{r} r
$$

where $M=\left(A_{z}+B_{z} K_{z}\right)^{-1}$.

To actively reject the effect of the disturbance, suppose $K_{d}$ satisfies $C_{z} M\left(B_{d}+B_{z} K_{d}\right)=0$. The disturbance compensation gain $K_{d}$ can be obtained from (8):

$$
K_{d}=-\left(C_{z} M B_{z}\right)^{-1} C_{z} M B_{d}
$$


Then by eliminating the disturbance effect from (7), the tracking problem can be solved if the feedforward gain $F_{r}=-\left(C_{z} M B_{z}\right)^{-1}$. For the boiler-turbine unit, it is easy to verify that $C_{z} M B_{z}$ is invertible and the design of the controller gain in (5) have been completed.

By taking the Laplace transformation of (4) and the control law (5), yields

$$
\begin{aligned}
& s \hat{\bar{Z}}(s)=\bar{A}_{z} \hat{\bar{Z}}(s)+\bar{B}_{z} U(s)+L\left(Y(s)-\bar{C}_{z} \hat{\bar{Z}}(s)\right) \\
& U(s)=K \hat{\bar{Z}}(s)+F_{r} R(s)
\end{aligned}
$$

where $K=\left[\begin{array}{ll}K_{z} & K_{d}\end{array}\right]$.

We obtain the following control structure

$$
U(s)=C_{1}(s) Y(s)+C_{2}(s) F_{r} R(s)
$$

where

$C_{1}(s)=\left[I-K\left(s I-\bar{A}_{z}+L \bar{C}_{z}\right)^{-1} \bar{B}_{z}\right]^{-1} K\left(s I-\bar{A}_{z}+L \bar{C}_{z}\right)^{-1} L$ and $C_{2}(s)=\left[I-K\left(s I-\bar{A}_{z}+L \bar{C}_{z}\right)^{-1} \bar{B}_{z}\right]^{-1}$.

It is clear to see that the controller is a two degree-of-freedom structure, where the tracking control and the feedback control is mutually independent. The second item of the controller represents the set point-filter respecting to the reference signal. The stability of the closed-loop system can be guaranteed by the first item of the controller.

\section{Multi-objective optimization}

Following the unit load demand ( $E_{\text {uld }}$ ) generated from the Automatic Generation Control (AGC) strictly while operating the plant in a safe and economical manner are the objectives for the control of boiler turbine unit. With the consideration of these factors, the multiobjective optimization can be brought to obtain the reference set-points under the linearized model plus the estimated lumped disturbance, in addition with various constraints.

Due to the estimation of $\hat{d}(t)$, the multi-objective optimization problem will be a linear optimization which can be fast solved in real time. According to the augmented system (3), we have:

$$
\begin{gathered}
\min _{z_{s}, u_{s}} J=\beta_{1} J_{1}\left(z_{s}, u_{s}\right)+\beta_{2} J_{2}\left(z_{s}, u_{s}\right)+\cdots+\beta_{k} J_{k}\left(z_{s}, u_{s}\right) \\
\text { s.t. } 0=A_{z} z_{s}+B u_{s}+L_{p}(s) \hat{d}(t) \\
u_{\min } \leq u_{s} \leq u_{\max } \\
y_{\min } \leq C_{z} z_{s} \leq y_{\max }
\end{gathered}
$$

where the objective functions $\left(J_{1}, J_{2} \cdots J_{k}\right)$ can be specified with their respective weights $\left(\beta_{1}, \beta_{1} \cdots \beta_{k}\right)$ for the optimization.

In this paper, we take the following objective functions for the boiler-turbine unit:

$$
J_{1}\left(z_{s}, u_{s}\right)=u_{s, 1}, J_{2}\left(z_{s}, u_{s}\right)=-u_{s, 3}, J_{3}\left(z_{s}, u_{s}\right)=\left|h_{s}-h_{\text {typical }}\right| \text { and } J_{4}\left(z_{s}, u_{s}\right)=\left|P_{s}-P_{t y p i c a l}\right|
$$

where $J_{1}$ represents the coal consumption from the fuel command, $J_{2}$ represents the pressure drop from the main steam valve where the throttle loss will decrease the efficiency of the system, $J_{3}$ represents the separator enthalpy deviation where the $h_{s}\left(y_{2}\right)$ should be set to the typical working point $h_{t y p i c a l}$ as close as possible and the $J_{4}$ represents the main 
steam pressure deviation where the $P_{s}\left(y_{1}\right)$ should be set close to the moving typical working point $P_{\text {typical }}$ obtained from the curve fitting based on typical operating points, and the $h_{\text {typical }}$ can be obtained similar to $P_{\text {typical }}$.

A low-pass filter $L_{p}(s)=\omega_{b} / s+\omega_{b}$ which is advised to suppress the high-frequency disturbances. The high frequency disturbances may cause the variation of the set point, which may result in the resonance of the closed-loop system. Here, a low-order filter $L_{p}(s)=\omega_{b} / s+\omega_{b}$ is advised, in which the band $\omega_{b}=2 \mathrm{rad} / \mathrm{s}$ is chosen considering the characteristics of the closed-loop system.

\section{Simulations}

In this section, simulation studies are presented to show the performance of the proposed controller. The feedback gain $K_{z}$ of the proposed method is set through poles placement at $(-1.2,-0.30,-2.5,-0.07)$ point and the observer gain $L$ is obtained by placing the poles two times of that for the feedback gain.
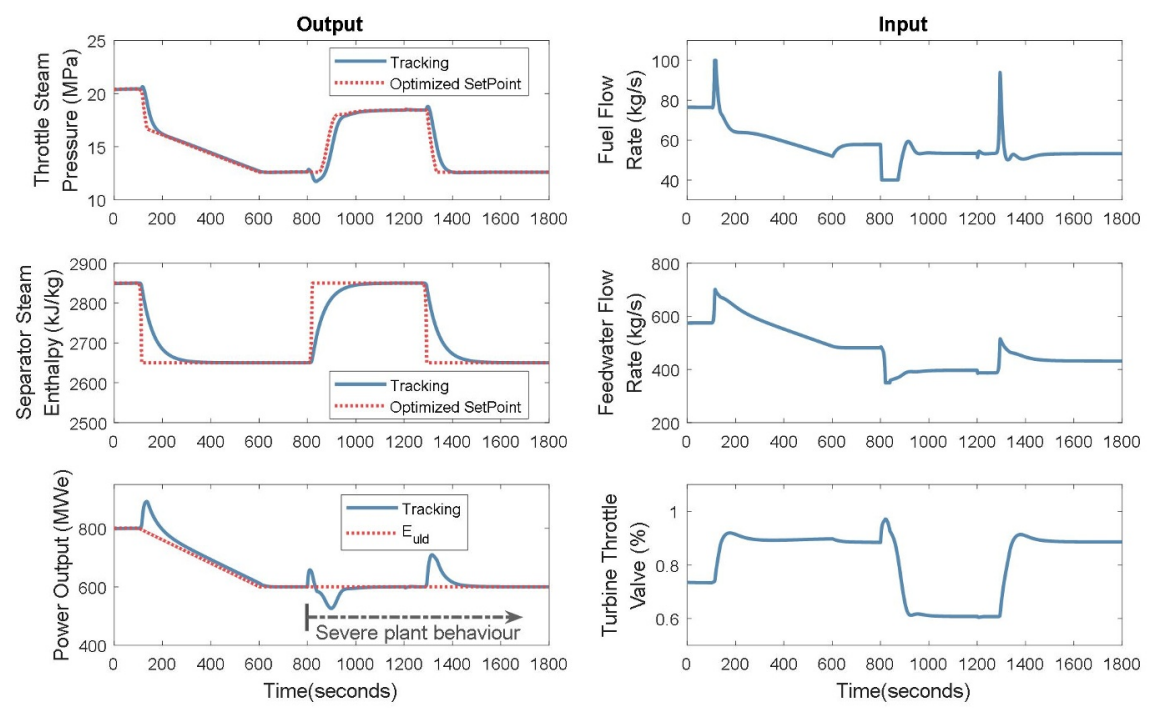

Fig. 2. Performance of multi-objective optimization with various disturbances.

Suppose that the boiler-turbine unit is operated at a steady-state point in which the set points are calculated at $E_{\text {uld }}=800 \mathrm{MWe}$, then at $t=100 \mathrm{~s}$, the input ( $E_{\text {uld }}$ ) of the multiobjective optimization is transferred from $800 \mathrm{MWe}$ to $600 \mathrm{MWe}$ at a rate of $24 \mathrm{MWe} / \mathrm{min}$. To illustrate the robustness of the control strategy, a severe model-plant mismatch condition is occurred in the simulation at $t=800 \mathrm{~s}$, where the parameters of plant (1) shrink to $85 \%$ of their original. Moreover, at $t=1300 \mathrm{~s}$, an external disturbance with magnitude $\Delta u_{2}=10 \mathrm{~kg} / \mathrm{s}$ is added on the feedwater flow.

The simulation as in figure 2 shows that the optimized set-points can be obtained to adapt the running condition when the plant behaviour is changed and/or large external disturbances happen, further, the multiple objectives can be reached at the tracking stage, 
such as the turbine throttle valve is kept as open as possible and the set-points of throttle steam pressure are obtained around $P_{\text {typical }}$ to guarantee the safety operation.

\section{Conclusions}

In this paper, a hierarchical control structure is proposed to optimize the control of the Ultra-Supercritical boiler-turbine unit. In the upper layer, the optimized set-points are calculated through a disturbance model by performing a high effective linear programming. In the lower layer, the feedthrough of the system is treated as a first-order dynamic augmented into the original disturbance model to reduce the complexity of the control design. Based on the augmented model, the states and the lumped disturbances are estimated by the MESO, where the lumped disturbances are filtered to formulate the upper disturbance model. The introduced low-pass filter can improve the feasibility of the multiobjective optimization. The simulation studies of the boiler-turbine show that the proposed method can achieve graceful performance with the consideration of multi-objective optimization and disturbance rejection.

\section{Acknowledgments}

The authors would like to acknowledge the National Natural Science Foundation of China (NSFC) under Grant 51506041, 51506029, the Natural Science Foundation of Jiangsu Province, China under Grant BK20150631.

\section{References}

1. Tan, Wen, et al. "Tuning of PID controllers for boiler-turbine units." ISA transactions 43.4 (2004): 571-583.

2. $\mathrm{Wu}$, J., et al. "Robust $\mathrm{H} \infty$ tracking control of boiler-turbine systems." ISA transactions 49.3 (2010): 369-375.

3. Chen, Pang-Chia, and Jeff S. Shamma. "Gain-scheduled $\ell 1$-optimal control for boilerturbine dynamics with actuator saturation." Journal of process control 14.3 (2004): 263-277.

4. Sun, Li, et al. "DEB-oriented modelling and control of coal-fired power plant." IFAC Proceedings Volumes 47.3 (2014): 413-418.

5. Han, Jingqing. "From PID to active disturbance rejection control." IEEE transactions on Industrial Electronics 56.3 (2009): 900-906.

6. Li, Shihua, et al. "Generalized extended state observer based control for systems with mismatched uncertainties." IEEE Transactions on Industrial Electronics 59.12 (2012): 4792-4802.

7. Garduno-Ramirez, Raul, and Kwang Y. Lee. "Multiobjective optimal power plant operation through coordinate control with pressure set point scheduling." IEEE Transactions on energy conversion 16.2 (2001): 115-122.

8. $\mathrm{Wu}, \mathrm{Xiao}$, et al. "Hierarchical optimization of boiler-turbine unit using fuzzy stable model predictive control." Control Engineering Practice 30 (2014): 112-123.

9. Liu, Ji-Zhen, et al. "A dynamic model used for controller design of a coal fired oncethrough boiler-turbine unit." Energy93 (2015): 2069-2078. 